\title{
TENSOR PRODUCTS OF POSITIVE MAPS OF MATRIX ALGEBRAS
}

\author{
ERLING STØRMER
}

\begin{abstract}
We give conditions for when tensor products of positive maps between matrix algebras are positive maps. Necessary and sufficient conditions are given.
\end{abstract}

\section{Introduction}

Even though positive linear maps appear in many situations in operator algebras and quantum information theory, main attention has so far been on completely positive maps. One reason for this is that tensor products of completely positive maps are positive, while this is false for general positive maps. In the present paper we shall consider the problem of when the tensor product of two positive maps is positive in the case when the underlying Hilbert spaces are finite dimensional.

It turns out that the problem is intimately related to symmetric mapping cones and their dual cones, see below for definitions. More specifically, if $\mathscr{C}$ is a symmetric mapping cone in $P(H)$ - the positive linear maps of the bounded operators $B(H)$ on $H$ into itself - then a map $\phi$ belongs to the dual cone $\mathscr{C}^{\circ}$ of $\mathscr{C}$ if if and only if $\psi \otimes \phi$ is positive for all $\psi \in \mathscr{C}$. Indeed, it suffices to know that $\psi \otimes \phi(p) \geq 0$, where $\frac{1}{n} p, n=\operatorname{dim} H$, is the density matrix for the maximally entangled state. As an application we show that if $K$ and $L$ are two other finite dimensional Hilbert spaces, and $\psi: B(K) \rightarrow B(H), \phi: B(L) \rightarrow B(H)$, then $\psi \otimes \phi$ is positive when $\psi$ is $\mathscr{C}$-positive, and $\phi$ is $\mathscr{C}^{\circ}$-positive.

We now recall the main concepts encountered in the sequel. By a mapping cone $\mathscr{C}$ we mean a closed subcone of $P(H)$ such that if $\phi \in \mathscr{C}$ and $\alpha, \beta \in$ $C P(H)$ - the completely positive maps in $P(H)$, then $\alpha \circ \phi \in \mathscr{C}$ and $\phi \circ \beta \in \mathscr{C}$. We say $\mathscr{C}$ is symmetric if $\phi \in \mathscr{C}$ implies both $\phi^{*} \in \mathscr{C}$ and $\phi^{t}=t \circ \phi \circ t \in \mathscr{C}$, where $\phi^{*}$ is the adjoint map of $\phi$ in the Hilbert-Schmidt structure on $B(H)$, viz. $\operatorname{Tr}(\phi(a) b)=\operatorname{Tr}\left(a \phi^{*}(b)\right)$ for $a, b \in B(H)$, and $t$ is the transpose map on $B(H)$ with respect to an orthonormal basis $e_{1}, \ldots, e_{n}$ for $H$, and $\operatorname{Tr}$ is the usual trace on $B(H)$.

\footnotetext{
Received 23 December 2010.
} 
If $\phi: B(K) \rightarrow B(H)$ then the functional $\widetilde{\phi}$ on $B(K) \otimes B(H)$ defined by

$$
\widetilde{\phi}(a \otimes b)=\operatorname{Tr}\left(\phi(a) b^{t}\right),
$$

plays an important role in the theory. For example, $\widetilde{\phi}$ is positive if and only if $\phi$ is completely positive [6]. By [7] and [8] its density matrix is the transpose $C_{\phi}^{t}$ of the Choi matrix $C_{\phi}$ for $\phi$, defined by

$$
C_{\phi}=\sum_{i, j=1}^{n} e_{i j} \otimes \phi\left(e_{i j}\right)=\iota \otimes \phi(p),
$$

where $\iota$ is the identity map and $p=\sum_{i j} e_{i j} \otimes e_{i j}$, and $\left(e_{i j}\right)$ is a complete set of matrix units for $B(H)$ such that $e_{i j} e_{k}=\delta_{j k} e_{i}$, see [1].

Let $\mathscr{C}$ be a mapping cone in $P(H)$. Then its dual cone is defined by

$$
\mathscr{C}^{o}=\left\{\phi \in P(H): \operatorname{Tr}\left(C_{\phi} C_{\psi}\right) \geq 0, \forall \psi \in \mathscr{C}\right\} .
$$

If $\mathscr{C}$ is symmetric, then by [9] $\mathscr{C}^{\circ}$ is also a symmetric mapping cone. We refer to the books [2] and [3] for the theory of completely positive maps.

Most of this work was done during a visit to Institute Mittag-Leffler (Djursholm, Sweden).

\section{The main results}

Let $\pi: B(H) \rightarrow B(H)$ be defined by $\pi(a \otimes b)=b^{t} a$. As in [8] Lemma 10 it follows by straightforward computation that if $\phi \in P(H)$ then

$$
\widetilde{\phi}=\operatorname{Tr} \circ \pi \circ\left(\iota \otimes \phi^{* t}\right) .
$$

In particular

$$
\widetilde{\iota}(x)=\operatorname{Tr} \circ \pi(x)=\operatorname{Tr}\left(C_{l} x\right)=\operatorname{Tr}(p x) .
$$

Thus

$$
\begin{aligned}
\widetilde{\phi}(x) & =\operatorname{Tr} \circ \pi\left(\iota \otimes \phi^{* t}(x)\right) \\
& =\operatorname{Tr}\left(p\left(\iota \otimes \phi^{* t}\right)(x)\right) \\
& =\operatorname{Tr}\left(\iota \otimes \phi^{t}(p) x\right) .
\end{aligned}
$$

Lemma 1. Let $\psi, \phi: B(H) \rightarrow B(H)$. Then

(i) $(\phi \circ \psi) \widetilde{(x)}=\operatorname{Tr}\left(\psi^{*} \otimes \phi^{t}(p) x\right), \forall x \in B(H \otimes H)$.

(ii) $\psi^{* t} \otimes \phi(p)=\iota \otimes(\phi \circ \psi)(p)$. 
Proof. By equations (1) and (2)

$$
\begin{aligned}
(\phi \circ \psi)(a \widetilde{\otimes} b) & =\operatorname{Tr} \circ \pi\left(\iota \otimes(\phi \circ \psi)^{* t}(a \otimes b)\right) \\
& =\operatorname{Tr} \circ \pi\left(a \otimes\left(\psi^{*} \circ \phi^{*}\left(b^{t}\right)\right)^{t}\right) \\
& =\operatorname{Tr}\left(a\left(\psi^{*} \circ \phi^{*}\right)\left(b^{t}\right)\right) \\
& =\operatorname{Tr}\left(\psi(a) \phi^{*}\left(b^{t}\right)\right) \\
& =\operatorname{Tr} \circ \pi\left(\psi(a) \otimes \phi^{* t}(b)\right) \\
& =\operatorname{Tr}\left(p \psi(a) \otimes \phi^{* t}(b)\right) \\
& =\operatorname{Tr}\left(\psi^{*} \otimes \phi^{t}(p)(a \otimes b)\right),
\end{aligned}
$$

proving (i). Using equations (1) and (2) we also have

$$
(\phi \circ \psi) \widetilde{(x)}=\operatorname{Tr}\left(p\left(\iota \otimes(\phi \circ \psi)^{* t}(x)\right)=\operatorname{Tr}\left(\iota \otimes(\phi \circ \psi)^{t}(p) x\right) .\right.
$$

Thus by (i) we have

$$
\psi^{*} \otimes \phi^{t}(p)=\iota \otimes \phi^{t} \circ \psi^{t}(p) .
$$

Since this holds for all $\phi$ and $\psi$, it also holds for all $\phi^{t}$ and $\psi^{*}$. Thus

$$
\psi^{* t} \otimes \phi(p)=\iota \otimes \phi \circ \psi(p),
$$

completing the proof of the lemma.

We can now prove our main result. Note that the equivalence (i) $\Leftrightarrow$ (ii) is also proved in [4].

TheOREM 2. Let $\phi \in P(H)$. Let $\mathscr{C}$ be a symmetric mapping cone in $P(H)$. Then the following conditions are equivalent.

(i) $\phi \in \mathscr{C}^{\circ}$ - the dual cone of $\mathscr{C}$,

(ii) $\phi \circ \psi$ is completely positive for all $\psi \in \mathscr{C}$,

(iii) $\psi \otimes \phi$ is positive for all $\psi \in \mathscr{C}$,

(iv) $\psi \otimes \phi(p) \geq 0$ for all $\psi \in \mathscr{C}$, where $p$ is as before the maximal entangled state.

Proof. Clearly (iii) implies (iv). Since $\phi \circ \psi$ is completely positive if and only if $\iota \otimes \phi \circ \psi(p) \geq 0$, by Lemma $1 \phi \circ \psi$ is completely positive if and only if $\psi^{* t} \otimes \phi(p) \geq 0$. Since $\mathscr{C}$ is symmetric, $\psi \in \mathscr{C}$ if and only if $\psi^{* t} \in \mathscr{C}$. Hence (ii) is equivalent to (iv). By [9] Thm. 2 a map belongs to $\mathscr{C}$ if and only if it is $\mathscr{C}$-positive. Hence by [8] Thm. $1, \phi \in \mathscr{C}^{o}$ if and only if $\psi^{t} \circ \phi$ is completely positive for all $\psi \in \mathscr{C}$. Since $\mathscr{C}$ is symmetric this holds if and only if $\psi \circ \phi$ is completely positive for all $\psi \in \mathscr{C}$, hence if and only if $\phi^{*} \circ \psi^{*}=(\psi \circ \phi)^{*}$ is 
completely positive for all $\psi^{*} \in \mathscr{C}$, hence if and only if $\phi^{*} \circ \psi$ is completely positive for all $\psi \in \mathscr{C}$. But $\mathscr{C}^{\circ}$ is symmetric by [9], Thm. 1 , so $\phi \in \mathscr{C}^{\circ}$ if and only if $\phi^{*} \in \mathscr{C}^{o}$. Thus (i) $\Leftrightarrow$ (ii).

It remains to show (i) implies (iii). For this let $\left(e_{i}\right)$ be an orthonormal basis for $H$ such that $e_{i j} e_{j}=e_{i}$, so $\frac{1}{n} p$ is the projection onto the subspace spanned by $\sum_{i} e_{i} \otimes e_{i}$. Let $x \in H \otimes H$. Then $x=\sum_{i} e_{i} \otimes x_{i}$ with $x_{i} \in H$. Then there is $v \in B(H)$ such that $v e_{i}=x_{i}$, hence $1 \otimes v\left(\sum_{i} e_{i} \otimes e_{i}\right)=x$. Let $q$ be the projection onto $C x$. Then it follows that $\operatorname{Ad}(1 \otimes v)(p)=\lambda q$ for some $\lambda>0$.

We have just shown that given a 1-dimensional projection $q \in B(H)$ there exists $v \in B(H)$ such that

$$
1 \otimes \operatorname{Ad} v\left(\frac{1}{n} p\right)=q
$$

Assuming (i) $\phi \circ \operatorname{Ad} v \in \mathscr{C}^{o}$, since $\mathscr{C}^{o}$ is a mapping cone. By Lemma 1

$$
\psi^{* t} \otimes(\phi \circ \operatorname{Ad} v)(p)=\iota \otimes \phi(\operatorname{Ad} v \circ \psi)(p) .
$$

Since $\operatorname{Ad} v \circ \psi \in \mathscr{C}$, by the equivalence of (i) and (ii) $\phi \circ \operatorname{Ad} v \circ \psi$ is completely positive, hence

$$
\iota \otimes \phi \circ \operatorname{Ad} v \circ \psi(p) \geq 0 .
$$

Thus by the choice of $v, \psi^{* t} \otimes \phi(q) \geq 0$. Since $q$ is an arbitrary 1-dimensional projection, $\psi^{* t} \otimes \phi$ is positive. Again, since $\mathscr{C}$ is symmetric, $\psi \otimes \phi$ is positive for all $\psi \in \mathscr{C}$. Thus (i) implies (iii), and the proof is complete.

Recall that a map $\phi: B(K) \rightarrow B(H)$ is $\mathscr{C}$-positive for a mapping cone $\mathscr{C}$ if the functional $\widetilde{\phi}$ is positive on the cone $\{x \in B(K \otimes H): \iota \otimes \alpha(x) \geq 0, \forall \alpha \in$ $\mathscr{C}$ ]. By [9], Thm. 2 or [6], Thm. 3.6, this is equivalent to $\phi$ belonging to the cone generated by maps of the form $\alpha \circ \beta$ with $\alpha \in \mathscr{C}$ and $\beta: B(K) \rightarrow B(H)$ completely positive. Recall from [9], Thm. 1, that if $\mathscr{C}$ is symmetric, so is $\mathscr{C}^{\circ}$. Using these facts we can extend the implication (i) $\Rightarrow$ (iii) in Theorem 2 to the following more general case.

Corollary 3. Let $K, L$ and $H$ be finite dimensional Hilbert spaces. Let $\mathscr{C}$ be a symmetric mapping cone in $P(H)$. Suppose $\psi: B(K) \rightarrow B(H)$ is $\mathscr{C}$-positive, and $\phi: B(L) \rightarrow B(H)$ is $\mathscr{C}^{\circ}$-positive. Then $\psi \otimes \phi: B(K \otimes L) \rightarrow$ $B(H \otimes H)$ is positive.

Proof. By the above discussion it suffices to show the corollary for $\psi$ and $\phi$ of the form $\psi=\alpha \circ \beta, \alpha \in \mathscr{C}, \beta: B(K) \rightarrow B(H)$ completely positive, and $\phi=\gamma \circ \delta$ with $\gamma \in \mathscr{C}^{\circ}, \delta: B(L) \rightarrow B(H)$ completely positive. Thus

$$
\psi \otimes \phi=(\alpha \otimes \gamma) \circ(\beta \otimes \delta),
$$


is positive, since $\beta \otimes \delta$ is completely positive and $\alpha \otimes \gamma$ is positive by Theorem 2. The proof is complete.

REMARK. If $\psi: B\left(K_{1}\right) \rightarrow B\left(H_{1}\right)$ is $k$-positive, i.e., $\psi \in P_{k}$ in the notation of [5], and $\phi: B\left(K_{2}\right) \rightarrow B\left(H_{2}\right)$ is $k$-superpositive, i.e., $\phi \in S P_{k}$ is of the form $\sum_{i}$ Ad $V_{i}, V_{i}: K_{2} \rightarrow H_{2}$, then they remain the same as maps into $B(H)$ if $H$ is a Hilbert space containing $H_{1}$ and $H_{2}$ as subspaces. Since $P_{k}^{o}=S P_{k}$, see e.g. [5], it follows from Corollary 3 that $\psi \otimes \phi$ is positive.

In Theorem 2 it is sometimes enough to consider only one map $\psi \in \mathscr{C}$ to conclude that $\phi \in \mathscr{C}^{\circ}$. The next corollary is of this type.

Corollary 4. Let $\psi \in P(H)$ satisfy $\psi=\psi^{*}=\psi^{t}$. Let $\mathscr{C}$ denote the mappng cone generated by $\psi$. Let $\phi \in P(H)$. Then $\phi \in \mathscr{C}^{\circ}$ if and only if $\psi \otimes \phi$ is positive.

Proof. $\mathscr{C}$ is generated as a cone by maps of the form $\operatorname{Ad} u \circ \psi \circ \operatorname{Ad} v$, so the assumptions on $\psi$ imply that $\mathscr{C}$ is a symmetric mapping cone. Since

$\operatorname{Ad} u \circ \psi \circ \operatorname{Ad} v \otimes \phi=(\operatorname{Ad} u \otimes \iota) \circ(\psi \otimes \phi) \circ(\operatorname{Ad} v \otimes \iota)$,

and $\operatorname{Ad} u \otimes \iota$ and Ad $v \otimes \iota$ are positive maps, it follows that $\alpha \otimes \phi$ is positive for all $\alpha \in \mathscr{C}$ if and only if $\psi \otimes \phi$ is positive, hence by Theorem $2, \phi \in \mathscr{C}^{\circ}$ if and only if $\psi \otimes \phi$ is positive, proving the corollary.

REMARK. Theorem 2 and Corollary 4 contain well known characterizations of completely maps. It $\psi=\iota$ then it satisfies the conditions of Corollary 4 , so the mapping cone $\mathscr{C}$ generated by $\psi$ is the cone of completely positive maps. Hence if $\phi \in P(H)$, then by Corollary $4, \phi \in \mathscr{C}^{\circ}$ if and only if $\iota \otimes \phi$ is positive, if and only if $\phi \in \mathscr{C}$ by definition of $\mathscr{C}$, so $\mathscr{C}^{\circ}=\mathscr{C}$. By Theorem 2 we have

$$
\begin{aligned}
C_{\phi}=\iota \otimes \phi(p) \geq 0 & \Leftrightarrow(\alpha \otimes \iota)(\iota \otimes \phi(p)) \geq 0, \forall \alpha \in \mathscr{C} \\
& \Leftrightarrow \alpha \otimes \phi(p) \geq 0, \forall \alpha \in \mathscr{C} \\
& \Leftrightarrow \phi \in \mathscr{C}^{o}=\mathscr{C} .
\end{aligned}
$$

In Corollary 4 we assumed $\psi=\psi^{*}=\psi^{t}$. These conditions can be easily verified by checking the corresponding conditions for the Choi matrix. The next proposition is also true for self-adjoint linear maps.

Proposition 5. Let $\phi \in P(H)$. Then $\phi=\phi^{*}=\phi^{t}$ if and only if $C_{\phi}$ is a real symmetric matrix invariant under the flip $a \otimes b \rightarrow b \otimes a$ on $B(H) \otimes B(H)$.

Proof. Let $J$ be the conjugation on $H \otimes H$ defined by

$$
J z e_{i} \otimes e_{j}=\bar{z} e_{j} \otimes e_{i}, \quad z \in \mathrm{C}
$$


where $e_{i}, \ldots, e_{n}$ is an orthonormal basis such that $e_{i j} e_{k}=\delta_{j k} e_{i}$. Then an easy computation shows that if $a, b$ are real matrices in $B(H)$, then $J a \otimes b J=b \otimes a$, so for $x \in B(H \otimes H)$, a real matrix with respect to the basis $\left(e_{i} \otimes e_{j}\right)$ for $H \otimes H$, then $x \rightarrow J x J$ is the flip $F$ applied to $x$.

We have $C_{\phi^{t}}=C_{\phi}^{t}$, so $\phi=\phi^{t}$ if and only if $C_{\phi}=C_{\phi}^{t}$, i.e., $C_{\phi}$ is symmetric. Since $\phi \in P(H), C_{\phi}$ is self-adjoint, hence $C_{\phi}$ is symmetric if and only if $C_{\phi}$ is real symmetric. Hence $\phi=\phi^{t}$ if and only if $C_{\phi}$ is real symmetric. By [9], Lem. 3, $C_{\phi^{*}}=J C_{\phi} J$. Hence $\phi=\phi^{*}=\phi^{t}$ if and only if $C_{\phi}$ is real symmetric, and by the first part of the proof, $C_{\phi}=F\left(C_{\phi}\right)$, so invariant under the flip, completing the proof.

Example. A specific example of a map as in Proposition 5 is given by $\phi=\operatorname{Ad} V$, where $V$ is a real symmetric matrix. Indeed, for general $V$ we have the formulas:

$$
(\operatorname{Ad} V)^{*}=\operatorname{Ad} V^{*}, \quad(\operatorname{Ad} V)^{t}=\operatorname{Ad} \bar{V},
$$

where $\bar{V}=\left(\overline{a_{i j}}\right)$ if $V=\left(a_{i j}\right)$, and $\operatorname{Ad} V(x)=V x V^{*}$. Thus, if $V$ is real symmetric, then $\operatorname{Ad} V=(\operatorname{Ad} V)^{*}=(\operatorname{Ad} V)^{t}$. Furthermore, if $V$ is real symmetric and $F$ the flip then

$$
C_{\mathrm{Ad} V}=\sum_{k l} e_{k l} \otimes \operatorname{Ad} V\left(e_{k l}\right)=\sum_{i j k l} v_{k i} v_{l j} e_{k l} \otimes e_{i j} .
$$

Thus

$$
\begin{aligned}
F\left(C_{\mathrm{Ad} V}\right) & =F\left(\sum_{i j k l} v_{k i} v_{l j} e_{k l} \otimes e_{i j}\right) \\
& =\sum_{i j k l} v_{k i} v_{l j} e_{i j} \otimes e_{k l} \\
& =\sum_{i j k l} v_{i k} v_{j l} e_{k l} \otimes e_{i j} \\
& =\sum_{i j k l} v_{k i} v_{l j} e_{k l} \otimes e_{i j} \\
& =C_{\mathrm{Ad} V},
\end{aligned}
$$

where we at the third equality sign changed the roles of $i$ and $k$, and $l$ and $j$, and used that $V$ was symmetric at the fourth. It follows that $C_{\mathrm{Ad} V}$ is invariant under the flip. 


\section{REFERENCES}

1. Choi, M-D., Completely positive linear maps on complex matrices, Linear Algebra and Appl. 10 (1975), 285-290.

2. Effros, E., and Ruan, Z-J., Operator Spaces, London Math. Soc. Monographs (New Series) 23, Oxford Univ. Press, Oxford 2000.

3. Paulsen, V., Completely Bounded Maps and Operator Algebras, Cambridge Studies in Adv. Math. 78, Cambridge Univ. Press, Cambridge 2002.

4. Skowronek,Ł., Cones with a mapping cone symmetry in the finite-dimensional case, Linear Algebra Appl. 435 (2011), 361-370.

5. Skowronek, Ł., Størmer, E., and Życzkowski, K., Cones of positive maps and their duality relations, J. Math. Physics 50 (2009), 062106.

6. Størmer, E., Extension of positive maps into B( $\mathscr{H}$ ), J. Funct. Anal. 66 (1986), 235-254.

7. Størmer, E., Separable states and positive maps, J. Funct. Anal. 254 (2008), 2303-2312.

8. Størmer, E., Duality of cones of positive maps, Münster J. Math. 2 (2009), 299-309.

9. Størmer, E., Mapping cones of positive maps, Math. Scand. 108 (2011), 223-232.

DEPARTMENT OF MATHEMATICS

UNIVERSITY OF OSLO

0316 OSLO

NORWAY

E-mail: erlings@math.uio.no 